\title{
ON THE EQUARS ATTITUDE DYNAMICS AND CONTROL
}

\author{
Ijar M. da Fonseca* \\ Gilberto Arantes $\mathrm{Jr}^{+}$ \\ Himilcon C. Carvalho ${ }^{++}$ \\ E-mails: ijar@dem.inpe.br; arantes@dem.inpe.br; himilcon@aeb.gov.br \\ National Institute for Space Research- INPE, S. J. Campos, S.P., Brazil \\ Peter M. Bainum ${ }^{* *}$ \\ E-mail: pbainum@howard.edu \\ Howard University, Washington D.C.
}

\begin{abstract}
$\underline{\text { Abstract }}$
This work presents a preliminary study of the Brazilian Equatorial Atmosphere Research Satellite (EQUARS) attitude control subsystem (ACS). The satellite ACS requirements are dictated by the scientific experiments and the power supply needs. The scientific experiments require a three-axis attitude control subsystem in order to accomplish their pointing goals. In this paper the attitude control studies are conducted on the basis of the Linear Quadratic Gaussian regulator approach (LQG) to evaluate the control performance and the control effort during the satellite normal operational mode. The results indicate that the necessary actuators (reaction wheels) are available commercially on the shelf. The MATLAB ${ }^{\circledR} /$ Simulink has been used as a software tool to implement all the computer simulations and visual graphics generation.
\end{abstract}

\section{Introduction}

Copyright $\odot 2004$ by the International Astronautical Federation. All rights reserved.

Research supported by INPE and FAPESP (Brazil), and Howard University, Washington D.C.

P

${ }^{*}$ Professor and Control Engineer, Space Mechanics and Control Division, Brazilian Institute for Space Research, INPE, S.J. Campos, S.P., Brazil.

+ Graduate Student, Space Mechanics and Control Division, Brazilian Institute for Space Research, INPE, S.J. Campos, S.P., Brazil.

${ }^{++}$EQUARS Project Manager, National Institute for Space Research, INPE, S.J, Campos, S.P., Brazil.

** Distinguished Professor of Aerospace Engineering, Emeritus, Dept. of Mechanical Engineering, Howard University, Washington D.C.; Fellow AIAA, Fellow AAS, Member IAA.
The EQUARS is a $750 \mathrm{Km}$ low Earth Orbit (LEO) spacecraft. The orbit inclination is $20^{\circ} \pm 2^{\circ}$. The ACS includes 4 reaction wheels, one of them being $\mathrm{n}$ a skew symmetric configuration. This fourth wheel is redundant to prevent failure of any of the others. One of the three normal operational wheels (pitch) is a momentum wheel. A momentum wheel is a reaction wheel with the nominal angular momentum different from zero ${ }^{1}$. The pitch wheel is considered also as a source of torque to spin up the spacecraft up to the orbital rate since the Earth pointing is required 


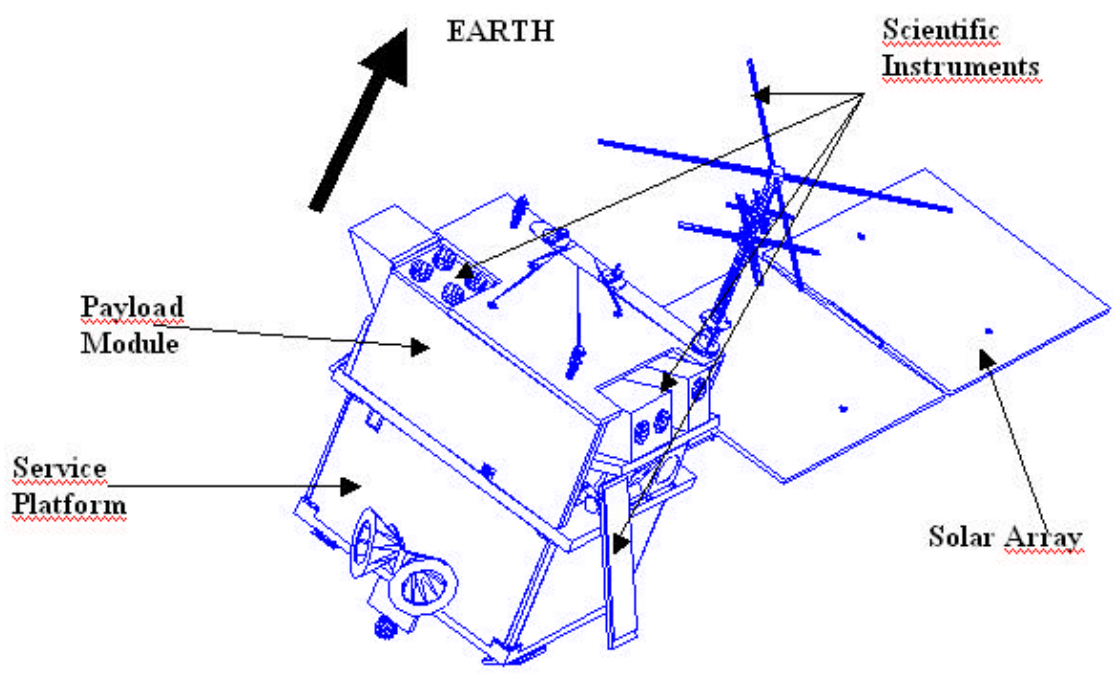

Fig. 1 - EQUARS structure and hardware

for the EQUARS mission. These actuators shall provide the necessary torque level for the spacecraft attitude control stabilization. Additional actuators are magnetic torquers, to provide the spacecraft with the necessary torque to de-saturate the wheels, when required. The torque coils will be used also for large maneuvers, when time requirements are not strict. A set of sensors (Sun, Star, Magnetometers, and Earth sensors) will complement the ACS hardware. The $120 \mathrm{Kg} 60 \times 60 \times 80 \mathrm{~cm}$ space vehicle in-orbit configuration (solar panel deployed) is shown in figure 1. The solar panel shall provide the satellite battery with enough energy during the mission lifetime.

The EQUARS scientific experiments involve integrated activities between Brazilian and researchers from other countries. Some of the experiments and the international associated institutions are shown in table 1

In order to satisfy the experiment requirements the satellite shall be Earth oriented while pointing toward the Earth limb. These pointing requirements impose a three-axis attitude control scheme for the satellite.

The satellite telecommunication system includes downlink data transmission. At least two ground stations are being considered to guarantee the communication with the spacecraft. The data flow and the downlink scheme is shown in figure 2

Table 1- International collaboration with the EQUARS scientific experiments

\begin{tabular}{|l|l|}
\hline Experiments & Institutions/Country \\
\hline GPS-OCCULT & $\begin{array}{l}\text { RASC/University of } \\
\text { Kyoto, Japan }\end{array}$ \\
\hline GWIM & UWO/Canada \\
\hline MLTM & USU/USA \\
\hline CERTO & NRL/USA \\
\hline
\end{tabular}




\section{The EQUARS ACS}

The purpose of the ACS is to provide the satellite with proper orientation and stability while pointing the satellite instruments and the solar panel. No orbit control is being considered for the EQUARS mission. However, the ACS subsystem performs on-board orbit determination from GPS position data, when available from the GPS scientific experiment. The ACS subsystem includes the on-board control software that shall work as an application in the on-board computer (OBC). The pointing requirements are:

- The satellite must be oriented such that some of scientific sensors point towards the limb of the Earth while a pointing device follows the local vertical.

- The pitch axis must be aligned with the orbit normal.

- The roll axis shall be aligned with the orbital velocity vector

- The third axis composes the dextrogyro orthogonal system.

The main attitude requirements are summarized in table 2.

The solar panel rotation will be Sun synchronized to guarantee the pointing requirements necessary for the satellite energy supply. A step motor will be used to accomplish these attitude and power supply requirements

Table 2: Attitude requirements

\begin{tabular}{|l|l|}
\hline Description & Specification \\
\hline Pointing & Nadir \\
$\begin{array}{l}\text { Nadir Pointing } \\
\text { accuracy }\end{array}$ & $0.5^{\circ}(3 \sigma)$ \\
\hline Stability & 0.015 Deg./sec \\
\hline Eccentricity & 0 \\
\hline $\begin{array}{l}\text { Attitude } \\
\text { knowledge }\end{array}$ & $0.05^{\circ}(3 \sigma)$ \\
\hline
\end{tabular}

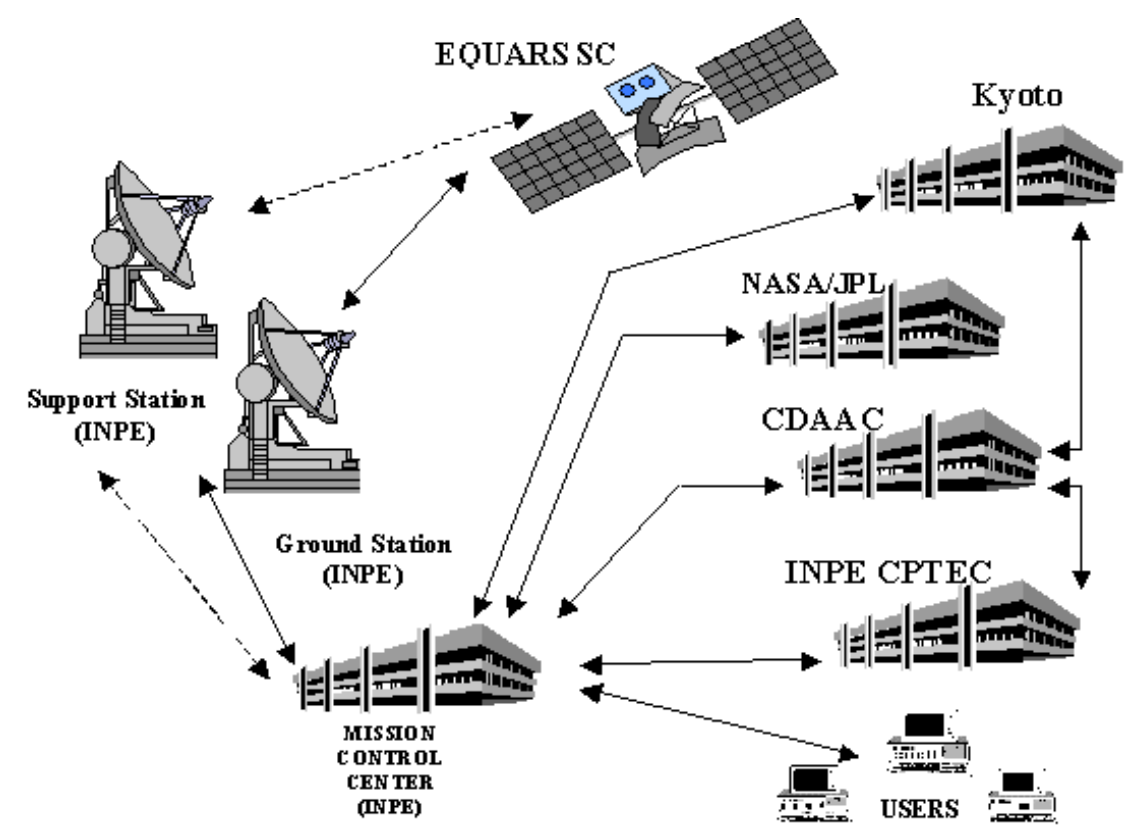

Fig. 2 -Donwlink Commnunication Schemme 


\section{$\underline{\text { ACS Operational Modes }}$}

Three modes are being considered for the EQUARS attitude control. They are

- Safe Mode: This operational mode shall deploy and point the solar panel towards the Sun and keep the vehicle in a stable safety condition. This mode shall also configure the satellite hardware to receive telecommands, by turning on the transmitters. The safe mode shall keep the instrument boresights and the battery face away from the Sun. The energy consumption shall be minimal when performing these tasks. Consistently with this requirement the spacecraft shall not provide electrical power to the

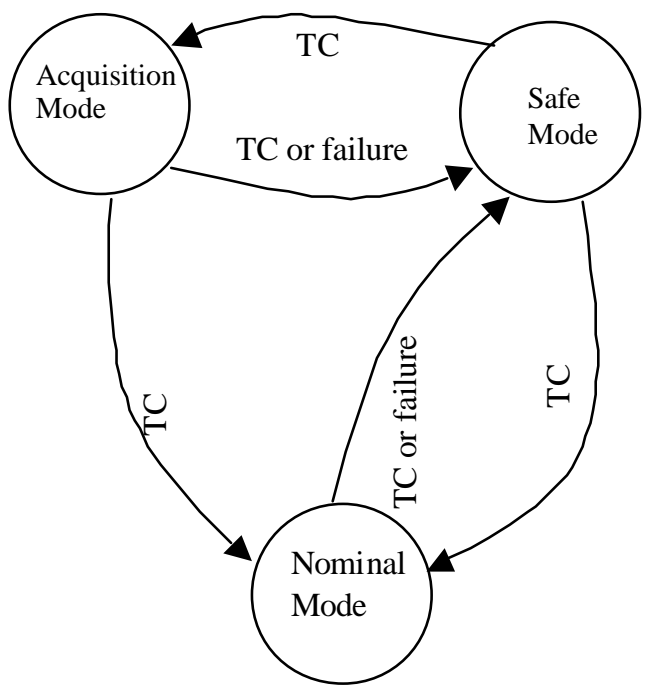

Fig. 3 - Modes Transition Diagram

Payload Module, except for thermal control, when required.

- Attitude Acquisition Mode: This is an intermediate mode, from safe mode to the nominal operational mode. At the end of this phase, the spacecraft shall be pointing to Nadir and the Payload equipment shall be off.

- Nominal Operational Mode: In this mode all the spacecraft equipment, including the Payload, are in their final operational configuration. The mission science data shall be collected and transmitted to the Earth. The spacecraft shall be pointed to the Nadir, and full pointing performance shall be maintained.

The transition between the modes is illustrated in figure 3. According to that diagram

- The transition from the Safe mode to other operational modes shall be done via telecommand only.

- The transition to the Safe Mode from the Nominal or Acquisition Modes shall occur in case of anomalies detected by either the ACS or the On Board Data Handling subsystem, OBDH.

- All the on-board operations during the Sun and Earth attitude acquisition phases shall be performed automatically.

Some of the ACS operating scenarios are discussed below.

- The Sun Acquisition (Safe Mode)

Upon separation, the solar panel is deployed, the transmitter is turned on to support the tracking by ground stations, and the satellite acquires a stable Sun pointing attitude, relying on a minimum set of equipment (magnetometer, Sun sensors, and magnetic torque coils). As the rotation rates fall below a given threshold, to allow the operation of the star tracker, the satellite is ready to receive commands to move to the next mode. A first check of all service 
platform equipment is performed before proceeding to Earth acquisition.

\section{- Earth Acquisition}

In this phase (Acquisition Mode), the on-board clock synchronization data and the orbital elements are uploaded from the ground to allow the on-board orbit propagation and Earth pointing operation by using a star tracker. Once the Earth pointing is acquired, the science instruments are checked, and the start of the Nominal Operational Mode will be commanded from the ground.

\section{- Nominal Operation}

In the nominal operation the EQUARS satellite undergoes a 7 day cycle, when it executes autonomously a Working Plan, composed by time-tagged commands, which control the operation of the onboard equipment and the science instruments for that period. The Working Plan spans through 9 days, to account for the ground segment outages. At the end of the 9 day period, without ground contact, the satellite enters in a stable, Sun pointing Safe Mode.

Within one orbit, the satellite shall collect science data and store them onboard for Earth downlink. Due to the orbit inclination and Ground Station locations, not all orbits provide contact opportunity. Seven among 15 successive orbits will be visible from the Ground Stations. During these visibilities the satellite receives commands, memory loading such as data files, software updates, ephemeredes, and timing corrections. The satellite transmits also real time and stored housekeeping telemetry (for health monitoring purposes) along with the science data. Memory dumps for diagnostics and monitoring purposes are also possible.

\section{The EQUARS Attitude Control Problem Formulation}

A three-axis attitude control scheme has been selected for the EQUARS. A satellite three-axis attitude control can be reached by using the Stabilite concept ${ }^{2}$. This concept requires just one momentum wheel and magnetic torquers to provide three-axis attitude control. Another option is to use either reaction wheels combined with torque coils or reaction wheels combined with jet gas. We have chosen a system of reaction wheels combined with torque coils for the EQUARS ACS.

Reaction wheels are essentially torque motors with high-inertia rotors ${ }^{1}$. Momentum wheels are reaction wheels with a nominal spin rate above zero to provide the vehicle with a nearly constant angular momentum. The momentum wheel spin-up generates the satellite pitch rate until its rotational motion is synchronized with the orbital rate. The associated angular momentum provides directional stability along the pitch axis while the roll and yaw axes are free to rotate once per orbit to guarantee the nadir pointing.

The external torque, when not cyclic, can saturate the reaction wheels since they absorb external disturbances. This fact justifies the need for the torque coils to de-saturate the wheels. The torque coils can also be used for large angle maneuvers in the attitude acquisition phase.

The EQUARS reaction wheels architecture is shown in figure 4. The fourth wheel is redundant and will be used in an emergency situation, for example, if one of the wheels fails .

The Euler approach ${ }^{3,4}$ can be used to obtain the equations of the dynamics. Following this approach, 
consider the total system angular momentum:

$$
\{H\}=\left\{H_{s}\right\}+\left\{H_{w}\right\}
$$

or

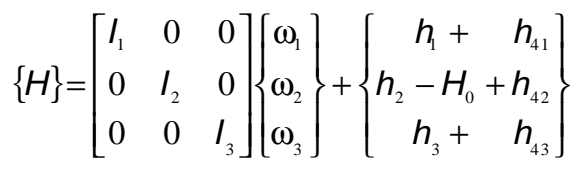

where $\left\{H_{w}\right\}$ accounts for the angular momentum of the four wheels. $h_{i}(i=1$ to 3) refers to the roll, pitch, and yaw reaction wheels angular momentum. $H_{0}$ refers to the nominal angular momentum of the pitch momentum wheel. The index $4 i \quad(i=1$ to 3$)$ indicates the components of the fourth wheel's angular momentum about the 1, 2, 3 axes. $\{\omega\}$ is the satellite angular velocity vector. The subscript $w$ means wheel. The external torque, $\{M\}$ is given by the rate of change in time of the total angular momentum. The non-linear system of equations is

$$
\{M\}=\{\dot{H}\}_{+}[\tilde{\omega}\}\{H\}=\left\{\dot{H}_{s}\right\}+\left\{\dot{H}_{w}\right\}+[\tilde{\omega}]\{H\}(3)
$$

where $\left\{\dot{H}_{w}\right\}$ is the reaction wheels

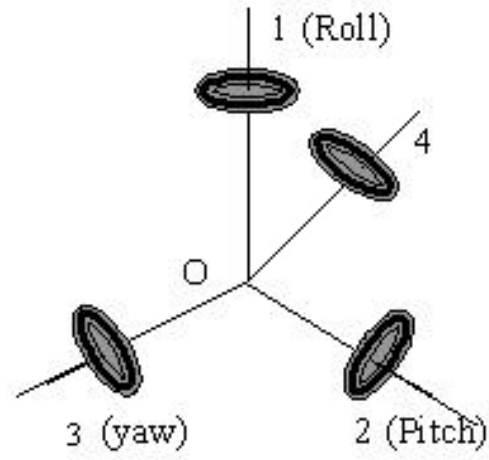

Fig. 4 - Four-reaction wheels configuration internal torque vector. This torque is used to change the satellite attitude when necessary but cannot be used to change the satellite total angular momentum. The momentum changes result from external torques. The external torque, when not cyclic, can saturate the reaction wheels since the wheels absorb this torque. This fact justifies the need for torque coils to de-saturate the wheels.

Let us consider the linear system equations given by Eq.(3) about the nominal attitude. Accounting for only small misalignments the angular velocity vector can be written as ${ }^{5}$

$$
\{\omega\}=\left\{\begin{array}{c}
\dot{\phi}-\omega_{0} \psi \\
\dot{\theta}-\omega_{0} \\
\dot{\psi}+\omega_{0} \phi
\end{array}\right\}
$$

where $\omega_{0}$ is the orbital rate.

Let us include the gravitygradient torque in this mathematical model for it is the most significant external perturbation at the satellite altitude of $750 \mathrm{Km}$. For small angles the components of the gravity-gradient toque along the satellite principal axes are $^{6}$.

$$
\left\{T_{g}\right\}=\left\{\begin{array}{c}
3 \omega_{0}^{2}\left(I_{3}-I_{1}\right) \phi \\
3 \omega_{0}^{2}\left(I_{3}-I_{2}\right) \theta \\
0
\end{array}\right\}
$$

\section{Control Law Formulation}

The Control law by using the Linear Quadratic Gaussian ${ }^{7}$ (LQG) state-space regulator is a modern control technique adopted for the EQUARS attitude control. This technique does not include the torque coil actuators, i.e., the optimal control law derived here includes only 
the four-reaction wheels architecture shown in figure 4. The objective is to regulate the output $y$ about zero. The plant is driven by the process measurement noise $\{w\}$ and the controls $\{u\}$. The regulator relies on the noise measurements $\left\{y_{v}\right\}=\{y\}+\{v\}$ to generate the controls. This procedure enables the designer to trade off regulation performance and control effort, and to take into account process and measurement noise. The LQG deals with a regulation problem as shown in figure 5 .

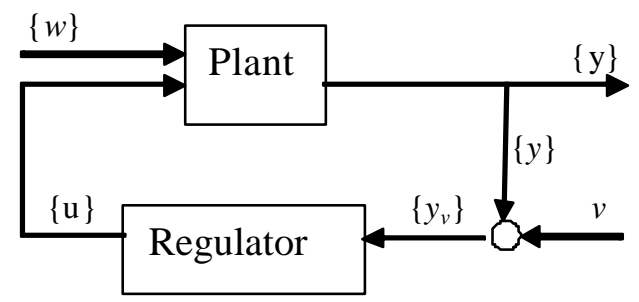

Figure 5 - The regulator problem

The LQG regulator consists of an optimal state-feedback gain and a Kalman state estimator. The optimal control problem can be stated as

Find the state-feedback control law

$$
\{u\}=-[F]\{x\}
$$

that minimizes the cost function

$$
I_{c}=\int_{0}^{\infty}\{x\}^{T}[Q]\{x\}+\{u\}^{T}[R]\{u\} d t
$$

$[Q]$ and $[R]$ are weighting matrices specified by the designer and define the trade-off between regulation performance (how fast $\{x\}$ goes to zero) and control effort. The optimal statefeedback gain matrix $[F]$ is obtained by solving the algebraic Riccati equation given $\quad \mathrm{b}[A]^{T}[P]+[P \llbracket A]+[Q]-[P\|B\| R]^{-1}[B]^{T}[P]=0$

where $[P]$ is the Riccati matrix, $[A]$ is the plant matrix.

The gain is called the LQ-optimal gain and is obtained here by the Linear Quadratic technique, LQR

The control law given by Eq.(6) cannot be implemented without full state measurements. However it is possible to derive a state estimation $\{\hat{x}\}$ such that the control

$\{u\}=-[F][\hat{x}\}$

remains optimal for the output-feedback problem. The state estimate is generated by the Kalman filter

$\{\dot{\hat{x}}\}=[A]\{\hat{x}\}+[B]\{u\}+[L]\left(\left\{y_{v}\right\}-[C]\{\hat{x}\}-[D]\{u\}\right)$

where $[L]$ is the Kalman gain. This is an optimal estimator when dealing with Gaussian white noise. It minimizes the asymptotic covariance

$\lim _{t \rightarrow \infty} E\left(\{x-\hat{x}\}\{x-\hat{x}\}^{T}\right)$

of the estimation error $\{e\}=\{x-\hat{x}\}$.

The LQG block linked to the Kalman filter is shown in figure 6 .

The LQG technique requires a state-space model for the plant. So let us define the state vector as 
$\{x\}=\left\{\begin{array}{c}\phi \\ \theta \\ \psi \\ \dot{\phi} \\ \dot{\theta} \\ \dot{\psi}\end{array}\right\}$

where the angles and the angle time derivatives stand for the attitude and attitude rates, respectively.

Then we can write the plant and the measurement equations as

$$
\{\dot{x}\}=[A]\{x\}+[B]\{u\}+[G]\{w\}
$$

$\left\{y_{v}\right\}=[C]\{x\}+[D]\{u\}+[H]\{w\}+\{v\}$

where $\{w\}$ and $\{v\}$ are modeled as white noise. The plant matrix is

$$
[A]=\left[\begin{array}{cccccc}
0 & 0 & 0 & 1 & 0 & 0 \\
0 & 0 & 0 & 0 & 1 & 0 \\
0 & 0 & 0 & 0 & 0 & 1 \\
a_{4,1} & 0 & 0 & 0 & 0 & a_{4,6} \\
0 & a_{5,2} & 0 & 0 & 0 & 0 \\
0 & 0 & a_{6,3} & a_{6,4} & 0 & 0
\end{array}\right]
$$

The elements of this matrix are defined as follows

$$
\begin{aligned}
& a_{4,1}=-\frac{4 \omega_{0}^{2}\left(I_{2}-I_{3}\right)+n H_{0}}{I_{1}} \\
& a_{4,6}=-\frac{\omega_{0}\left(I_{2}-I_{1}-I_{3}\right)-\omega_{0} H_{0}}{I_{1}} \\
& a_{5,2}=-\frac{3 \omega_{0}^{2}\left(I_{1}-I_{3}\right)}{I_{2}} \\
& a_{6,3}=-\frac{\omega_{0}^{2}\left(I_{2}-I_{1}\right)+\omega_{0} H_{0}}{I_{3}} \\
& a_{6,4}=-\frac{\omega_{0}\left(I_{1}-I_{2}+I_{3}\right)-\omega_{0} H_{0}}{I_{3}}
\end{aligned}
$$

The $[B]$ matrix is given by

$$
[B]=\left[\begin{array}{cccccccc}
0 & 0 & 0 & 0 & 0 & 0 & 0 & 0 \\
0 & 0 & 0 & 0 & 0 & 0 & 0 & 0 \\
0 & 0 & 0 & 0 & 0 & 0 & 0 & 0 \\
-\frac{1}{I_{1}} & 0 & 0 & -\frac{1}{I_{1} \sqrt{3}} & 0 & 0 & \frac{1}{I_{1}} & \frac{1}{I_{1} \sqrt{3}} \\
0 & -\frac{1}{I_{2}} & 0 & -\frac{1}{I_{2} \sqrt{3}} & 0 & 0 & 0 & 0 \\
0 & 0 & -\frac{1}{I_{3}} & -\frac{1}{I_{3} \sqrt{3}} & -\frac{1}{I_{3}} & 0 & 0 & -\frac{1}{I_{3} \sqrt{3}}
\end{array}\right]
$$

The control vector components can be written as

$$
\begin{aligned}
& u_{1}=\dot{h_{1}} \\
& u_{2}=\dot{h}_{2} \\
& u_{3}=\dot{h}_{3} \\
& u_{4}=\dot{h}_{4} \\
& u_{5}=\omega_{0} h_{1} \\
& u_{6}=\omega_{0} h_{2} \\
& u_{7}=\omega_{0} h_{3} \\
& u_{8}=\omega_{0} h_{4}
\end{aligned}
$$

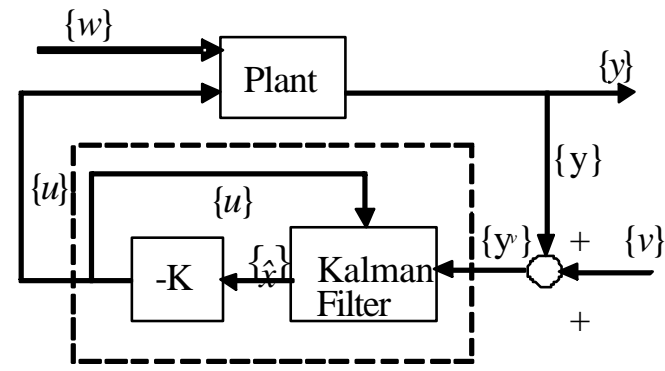

Figure 6- LQG regulator and the Kalman Filter

The control torques represented by the components $u_{i}, \quad(i=4, \ldots, 8)$ results from the gyroscopic coupling between the reaction wheel rotations and the angular velocity vector $\left\{\omega_{o}\right\}$,

The preliminary inertia matrix for the EQUARS is

$$
I=\left[\begin{array}{ccc}
13.31 & 0.37 & 0.39 \\
0.37 & 14.22 & -0.22 \\
0.39 & -0.22 & 11.2
\end{array}\right]
$$


When implementing the LQG procedure this matrix has been replaced by the associate principal moments of inertia.

The gain matrix $[F]$ has been obtained via the Linear Quadratic Regulator, LQR. For the input matrices $[R]$ and $[Q]$ given by<smiles>C[As]</smiles>

and

$\left[Q_{L Q R}\right]=\left[\begin{array}{cccccc}1 & & & & & \\ & 1 & & & 0 & \\ & & 1 & & & \\ & & & 1 & & \\ & 0 & & & 1 & \\ & & & & & 1\end{array}\right]$

the gain matrix is

$F=\left[\begin{array}{cccccc}0.2236 & 0 & 0.0008 & 1.5915 & 0 & 0 \\ 0 & 0.3162 & 0 & 0 & 2.9144 & 0 \\ -0.0008 & 0 & 0.2236 & 0 & 0 & 1.8054 \\ 0 & 0 & 0 & 0 & 0 & 0 \\ -0.0008 & 0 & 0.2236 & 0 & 0 & 1.8054 \\ 0 & 0 & 0 & 0 & 0 & 0 \\ -0.2236 & 0 & -0.0008 & -1.5915 & 0 & 0 \\ 0 & 0 & 0 & 0 & 0 & 0\end{array}\right]$

The gain matrix is useful for evaluation of some practical aspects of the control implementation. For example, if the gain matrix is diagonal or has the elements off the diagonal close to zero, it would be easier to implement the control gains in electrical systems, for instance. Otherwise it can be difficult to pass from theory to practical system implementation
The inputs for the Kalman filter are:

$R_{K}=\left[\begin{array}{cccccc}\sigma_{\text {roll }}^{2} & & & & & \\ & \sigma_{\text {pitch }}^{2} & & & 0 & \\ & & \sigma_{\text {yaw }}^{2} & & & \\ & & & \sigma_{\text {rateroll }}^{2} & & \\ & 0 & & & \sigma_{\text {ratepich }}^{2} & \\ & & & & & \sigma_{\text {rateyaw }}^{2}\end{array}\right]$ (20)

where:

$\sigma_{\text {roll }}=\sigma_{\text {pitch }}=\sigma_{\text {yaw }}=0.05$ degrees

$\sigma_{\text {rateroll }}=\sigma_{\text {ratepitch }}=\sigma_{\text {rateyaw }}=0.005 . \mathrm{deg} . / \mathrm{sec}$

$Q_{K}=\left[\begin{array}{cccccc}\sigma_{d}^{2} & & & & & \\ & \sigma_{d}^{2} & & & 0 & \\ & & \sigma_{d}^{2} & & & \\ & & & \sigma_{d}^{2} & & \\ & 0 & & & \sigma_{d}^{2} & \\ & & & & & \sigma_{d}^{2}\end{array}\right]$

where

$\sigma_{d}=1.5$

The covariance obtained was

$P=\left[\begin{array}{cccccc}0.2258 & 0 & 0 & 0 & 0 & 0 \\ 0 & 0.2285 & 0 & 0 & 0 & 0 \\ 0 & 0 & 0.2285 & 0 & 0 & 0 \\ 0 & 0 & 0 & 0.0228 & 0 & 0 \\ 0 & 0 & 0 & 0 & 0.0228 & 0 \\ 0 & 0 & 0 & 0 & 0 & 0.0228\end{array}\right] 10^{4}$

The results of the simulations in terms of the measurements, estimation, attitude, attitude rates, and control efforts are shown in figures 7 to 12 . Figures 7 and 8 compare the results for the attitude measurements and attitude estimation. These figures illustrate the good performance of the Kalman estimator. Figure 9 shows the estimate and measurements of the attitude angles (combined details of figure 7 and 8). The 
curves of large amplitude show details of figure 7 while embedded curves of smaller amplitude show details of figure 8. Figure 10 enhances the error estimation. Figures 11 and 12 illustrate the torque necessary to drive the system from attitude misalignments from about 5 degrees to zero. The torque shown in figures 11 to 12 is of different nature. Figure 11 deals with the torque imparted on the reaction wheels, while figure 12 shows the gyroscopic torque generated by the vector product of the orbital angular velocity vector and the wheels' angular momentum vectors. This feature of the dynamics can be observed in Eq. (3).

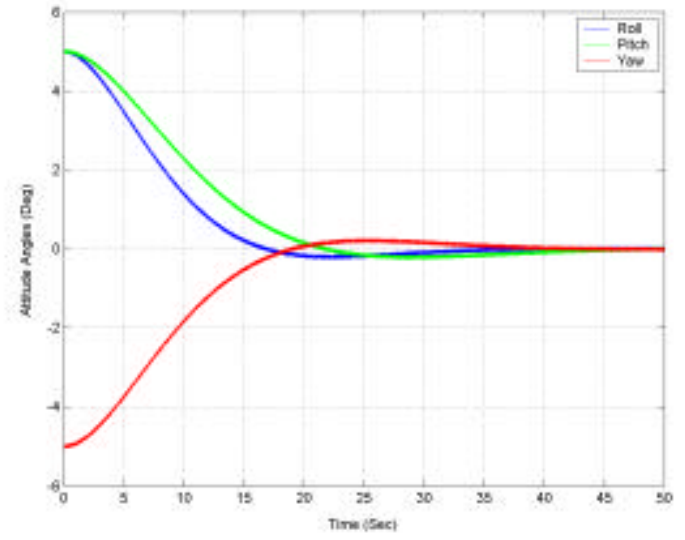

Fig. 7 - Attitude angle measurements \& time

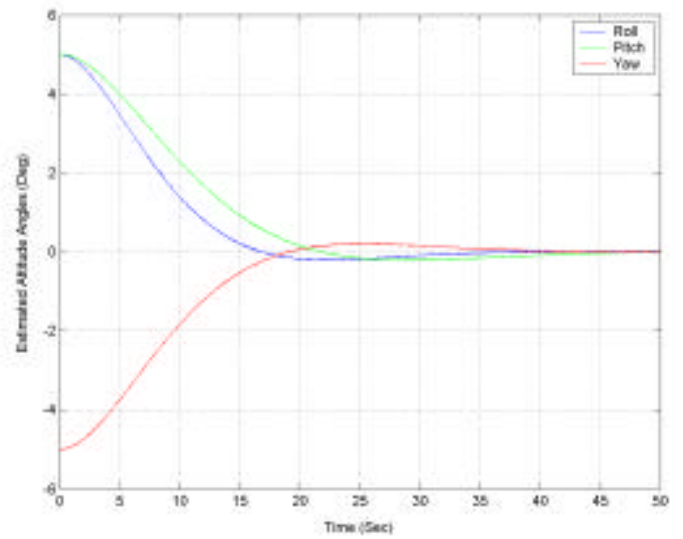

Fig. 8 - Attitude angle estimates $\&$ time

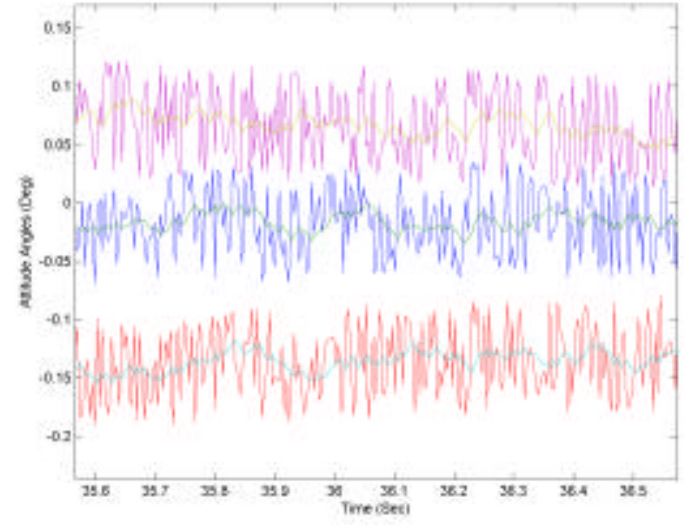

Fig. 9 - Estimate and measurements of the attitude angles (combined details of figures 7 and 8)

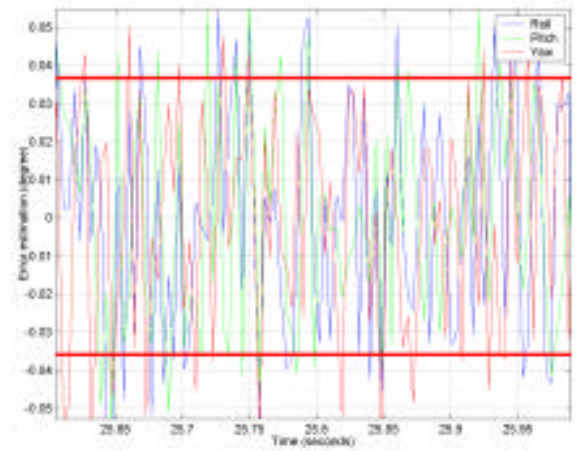

Fig.10 - Error estimation

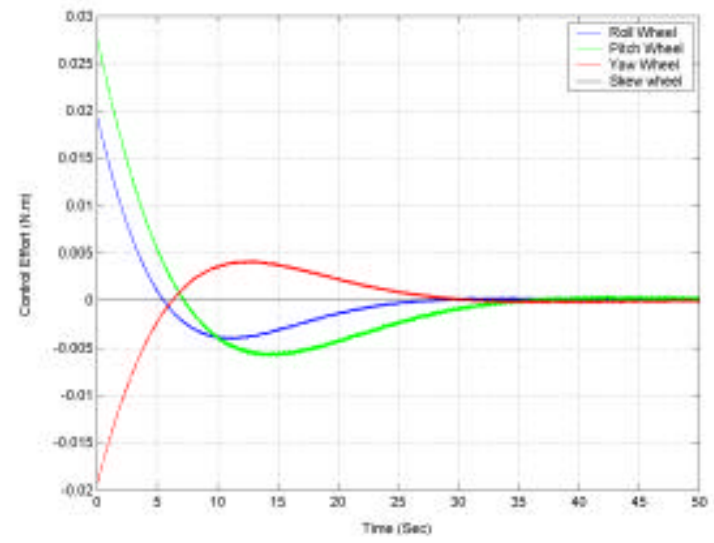

Fig. 11 - Control effort - wheels 


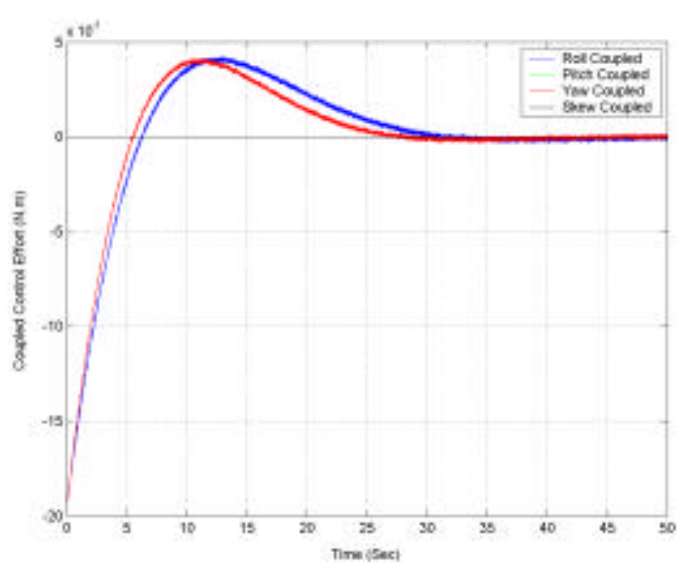

Fig. 12- Coupling control torques

The control effort shown in the above result is compatible with on the shelf reaction wheels $(0.030 \mathrm{N.m})$. This is important information for the EQUARS budget management. Another information we obtained from the simulation is that the reaction wheels working on the basis of the LQG technique procedure drives 5-degree attitude angle errors to zero in about 30 seconds.

\section{$\underline{\text { Conclusion }}$}

This work has presented
information about the EQUARS
scientific mission, about the hardware
(sensors and actuators) for the ACS, and
the operational control modes. The LQG
regulator is the option chosen to
implement the attitude control during the
normal operational mode. The EQUARS
attitude control problem formulation has
been developed taking into account the
architecture of four reaction wheels, one
of them working as a momentum wheel
and another one placed in a skew
symmetric configuration to prevent
failure of any one of the other three
wheels. The mathematical model has
been implemented in the
Matlab ${ }^{\circledR} /$ Simulink environment. The

results define the appropriate torque level that must be satisfied by the actuators to maintain the normal operational mode as a stable configuration.

\section{$\underline{\text { References }}$}

1. Larson, Wiley and Wertz, J. R.; Space Mission Analysis Design, Space Technology Library, Kluwer Academic Publishers, p. 352

2. Perkel, H.; Stabilite - A three -axis control system utilizing a single reaction wheel, AIAA Communications Satellite Systems Conference, Washington D. C. , May 2-4, 1966

3. Kaplan, M. H., Modern Spacecraft Dynamics \& Control, New York, John Willey \& Sons, 1976

4. Wertz, J. R.; Spacecraft Attitude Determination and Control, D. Reidel Publishing Company, England, 1978

5. Bryson, Arthur Jr;; Control of Spacecraft and Aircraft, Princeton University Press, 1994

6. Wie, B.; Space Vehicle Dynamics and Control, AIAA Education Series, 1998

7. Maciejowski, J.M.; Multivariable Feedback Design, Addison-Wesley Company, England, pp. 222-264, 1989 